\title{
Simulation and Optimization of Multi-step Stamping Forming Process for Car Front Panel
}

\author{
HUANG Zhao-ming, WANG Li
}

\begin{abstract}
Taking the front panel of a car as the research object, the whole process forming process plan of the parts is analyzed and planned. Based on this scheme, the initial sheet profile is calculated by one-step method. The whole process of stamping forming is simulated and analyzed based on AutoForm software and the forming defects are predicted and the forming process is optimized. The simulation and optimization results show that the whole process multi-step stamping simulation can obtain the process die surface quickly and effectively, and realize the seamless joint between the whole process die surface design and multi-step stamping simulation and optimization design. Reasonable product process optimization and trimming line optimization reduce the cracking phenomenon and actual boundary contour error.
\end{abstract}

Index Terms- whole process, initial sheet profile, forming process optimization, multi-step stamping forming, die surface design

\section{INTRODUCTION}

With the increasing complexity of body design and the requirement of surface accuracy and quality, many body panels need to be obtained by multi-process stamping ${ }^{[1]-[2]}$. The whole process stamping can effectively improve the quality and accuracy of the workpiece surface, but the forming mechanism of the wrinkling, springback and cracking of the workpiece surface becomes more and more complex. With the continuous development of computer design technology and simulation technology, the success rate of drawing forming simulation is greatly improved, and it also provides strong support for the implementation of multi-step stamping forming in the whole process ${ }^{[3]-[4]}$.

Relevant research shows that reasonable process planning, reasonable drawing die surface design including drawing process supplementary surface design, blank surface design, drawing rib design, application of drawing $\mathrm{rib}^{[5]-[7]}$, setting of drawing simulation process and optimization of forming process are the key factors in multi-step stamping process design ${ }^{[8]-[11]}$.

This paper takes car front panel as the research object and establishes the whole process stamping process plan, designs the whole process die surface and completes the numerical simulation and optimization of stamping process.

The purpose of this paper is to quickly feedback the forming problem of complex parts through CAE technology ${ }^{[12]-[15]}$.

HUANG Zhao-ming,Wentian College, Hohai University, Ma'anshan, People's Republic of China

WANG Li, XuanCheng Vocational \& Technical college, Xuancheng, People's Republic of China
It takes the uniformity of sheet thickness as the evaluation index, put forward the optimization scheme of forming process based on the satisfying the forming limit diagram after stamping, which can provide regularity for the automobile body die development and researcher.

\section{WHOLE PROCESS FOR PROCESS PLANNING}

The front panel of the car is left and right parts, and the shape is a half-box part with a convex platform. The three-dimensional digital model of the right panel is shown in Figure 1.For the shape of the part, the forming quality and production efficiency can be improved by combining the left and right parts of the opening side and forming the main surface of the part with the drawing process. In addition to the drawing process, the parts also include flanging, trimming and punching. It is necessary to plan the whole process plan when designing the die surface of the whole process. According to the engineering experience, the forming sequence of "OP05 blanking, OP10 primary drawing, OP20 secondary drawing, OP30 trimming and punching, OP40 flanging" is the most reasonable.

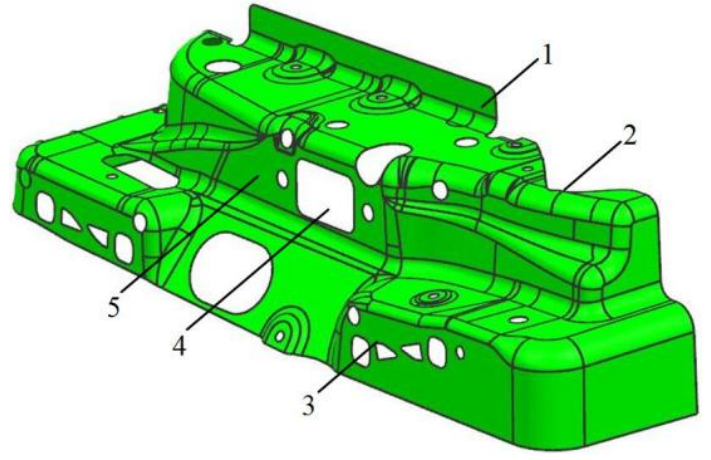

Fig.1 3D module of car rear panel

1-Flanging, 2-Cutting edge, 3-The second drawing, 4-Punching, 5-The first drawing

\section{WHOLE PROCESS FOR MOLD DESIGN}

\section{A. Whole Process for Die Surface Design}

Based on the above process plan, the drawing die surface can be designed quickly and effectively by using CAE whole driving method, which can improve the efficiency of die development and shorten the development cycle of the die. Firstly, the three-dimensional digital model of the right part of car front panel is imported into AutoForm software, and then parts preparation, flanging die surface design, line by $40 \mathrm{~mm}$ as the reference boundary line for solving the minimum sheet, defining the blank holder 
condition as the blank holder free-form sheet, and then using one-step solver to solve the problem. When the solution is completed, the boundary line is automatically imported into the current simulation file, the two-piece sheet expansion diagram of the front panel of the car is shown in Fig.2.

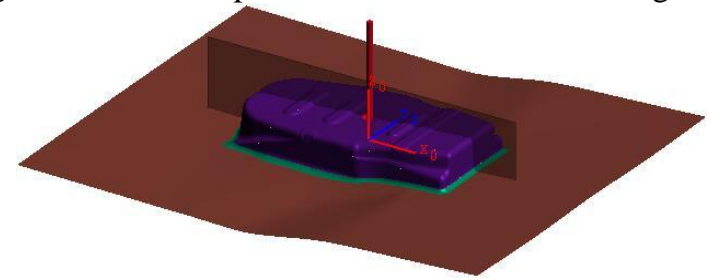

Fig.2 First drawing die surface-Draw20

\section{B. Sheet Development and Sheet Parameter Performance}

There are single step process based on single process and incremental method based on whole process for CAE driven chip expansion calculation. The first method in AutoForm is to use rapid prototyping simulation analysis calculation and sheet generator to solve the rough calculation of the smallest sheet, which is generally applied to the setting of forming simulation process. The second method is to optimize the profile of sheet metal by trimming line optimization module. It mainly aims at the accurate calculation of the whole process of sheet metal forming with high quality requirements. It is often used in the process optimization of simulation results.

\section{Sheet Expansion}

The material chip generator is used to solve the smallest chip in the die surface, and the parts are input by half symmetrical parts. The contour of the minimum sheet is solved by choosing the first drawing die surface which is shown in Fig. 2 as the object of solution in the application of the minimum sheet, using the expansion of the die parting line by $40 \mathrm{~mm}$ as the reference boundary line for solving the minimum sheet, defining the blank holder condition as the blank holder free-form sheet, and then using one-step solver to solve the problem. When the solution is completed, the boundary line is automatically imported into the current simulation file, the two-piece sheet expansion diagram of the front panel of the car is shown in Fig.3.

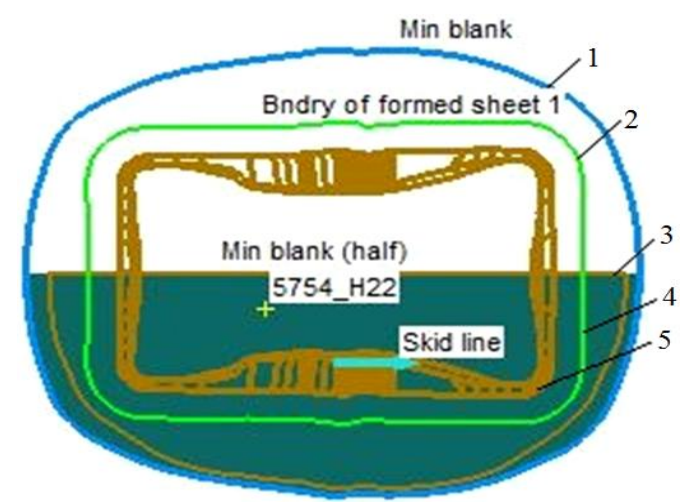

Fig3 Expansion drawing of two pieces of car front panel

1-Two pieces minimum profile, 2-Forming boundary of sheet material, 3-Right piece minimum material contour, 4-Sheet, 5-Slip line

\section{Sheet Parameter Performance}

The material used for the part is magnesium-aluminum alloy $5754 \mathrm{H} 22$, the thickness of the sheet is $1.2 \mathrm{~mm}$, and the control parameters are set as shell elements. The mechanical properties of the material are imported into the material library of AutoForm software from the material library for finite element numerical simulation. When the material is aluminum, Young modulus is $7 * 104 \mathrm{~N} / \mathrm{mm}^{2}$, Poisson's ratio is 0.30 , specific gravity is $2.7 * 105 \mathrm{~N} / \mathrm{mm}^{3}$, hardening curve is Hockett-Sherby, yield surface is Barlat, biaxial stress factor $\mathrm{M}=8.0$, forming limit curve is Arcelor V9 A1, corresponding hardening curve, yield surface and forming limit curve are shown in Fig. 4.

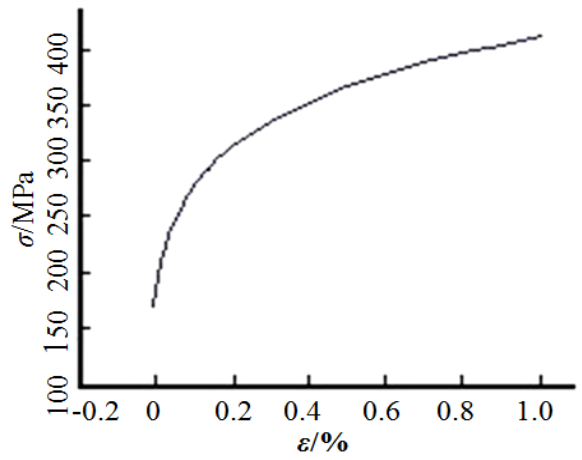

a)Hardening curve

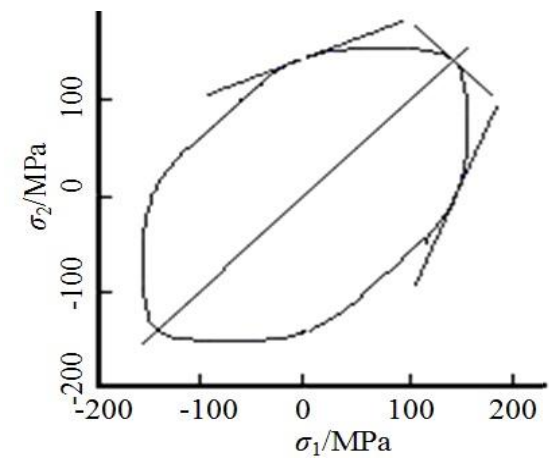

b)Yield surface

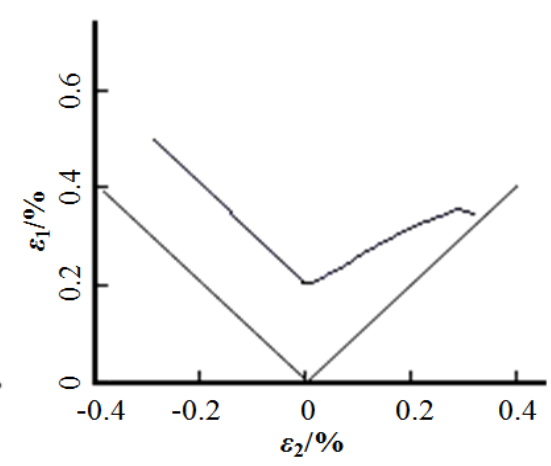

c)Forming limit curve

\section{Fig.4 Mechanical properties curve or surface of material $5754 \mathrm{H22}$}

\section{Whole Process for Process Design}

The sequence of the whole process design is opposite to that of the die surface design. The sequence of the whole process design of the front panel of a car is Draw20 process setting, Draw30 process setting, trimming punching and flanging Form50 process design. Each process mainly includes material sheet setting, die setting, lubrication setting, die motion process setting and control setting. The key factors of die motion process setting mainly include process steps and corresponding tool motion process or related parameters.

The first drawing of car front panel is single-action drawing, which requires not only setting drawing steps, but also setting gravity and closing steps. The motion process and related parameters of Draw20 tool are listed in Table I . The second drawing is completed on the basis of the first drawing, so it is necessary to shape the part of the first drawing reliably. The process includes four steps: positioning, first closing, second closing and drawing. Its Draw30 tool movement process and related parameters are listed in Table II. The punching and flanging process includes two steps: closing and flanging. The motion process and related parameters of Draw50 tool are listed in Table III. The corresponding finite element model of the 
World Journal of Research and Review (WJRR) ISSN:2455-3956, Volume-7, Issue-5, November 2018 Pages 01-05

whole process is shown in Fig.5(a), (b), (c).The numerical simulation results of multi-step stamping forming in the whole process are shown in Fig.6(a). Rainbow diagram shows that there is a crack in the part. Fig.6 (c) shows that the actual boundary outline of the part has a large error with the target boundary outline, it means process optimization is needed.

Tablel Tool Movement Process and Related Parameters of Draw20

\begin{tabular}{cccc}
\hline Work step & Concave die & Punch & Edge ring \\
\hline Gravity & Not active & Not active & Static \\
Close & $+500 \mathrm{~mm}$ & Not active & Static \\
Drawing & $+500 \mathrm{~mm}$ & Static & 1.5 ton \\
\hline
\end{tabular}

Tablell Tool Movement Process and Related Parameters of Draw30

\begin{tabular}{lllll}
\hline Work step & Concave die & $\begin{array}{l}\text { Punc } \\
\mathrm{h}\end{array}$ & Pressure plate & Edge ring \\
\hline Location & Not active & Static & Not active & Static \\
Primary closure & Not active & Static & $+500 \mathrm{~mm}$ & Static \\
Second closure & $+621 \mathrm{~mm}$ & Static & $3 \mathrm{MPa}$ & Static \\
Drawing & $+79 \mathrm{~mm}$ & Static & $3 \mathrm{MPa}$ & 1.5 ton \\
\hline
\end{tabular}

Tablelll Tool Movement Process and Related Parameters of Draw50

\begin{tabular}{llll}
\hline Work step & Support block & Flanging block & Press plate \\
\hline Closure & Static & Not active & $+500 \mathrm{~mm}$ \\
Flanging & Static & $+500 \mathrm{~mm}$ & $3 \mathrm{MPa}$ \\
\hline
\end{tabular}
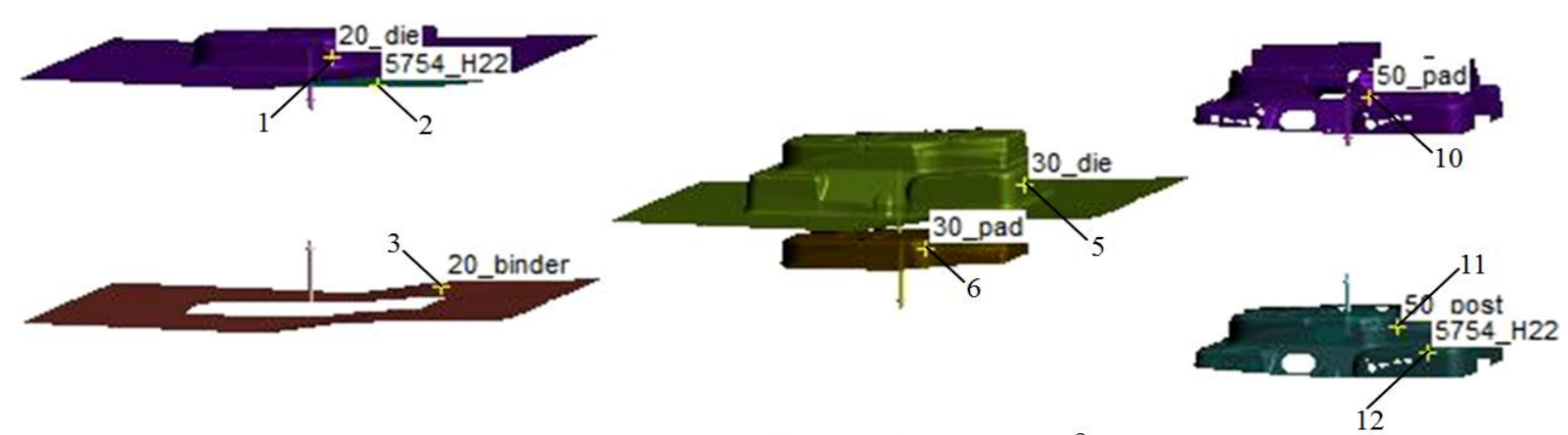

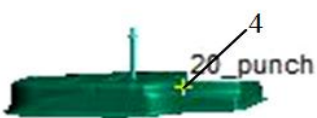

a)The first drawing process

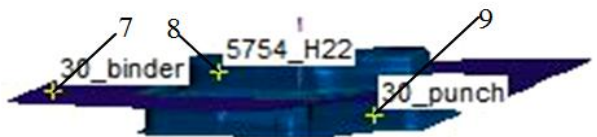

b)The second drawing process

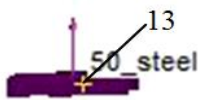

c)Flanging process

Fig.5 Whole Process for Finite Element Model of Car Front Panel

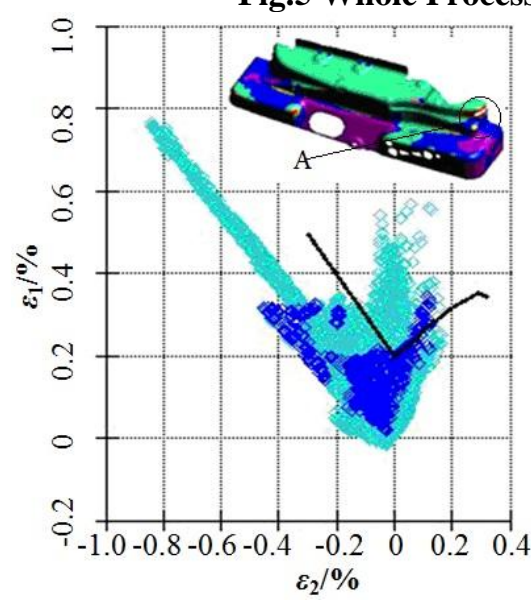

a)Before cracking optimization

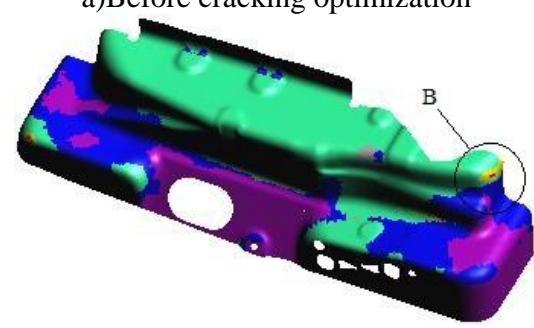

b)After cracking optimization

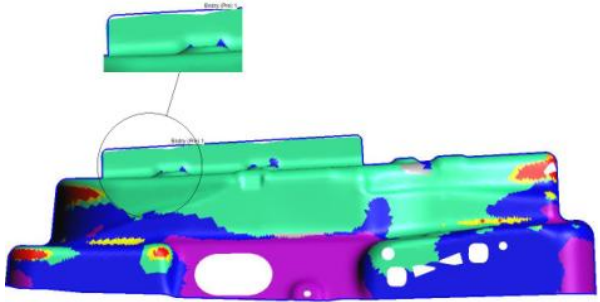

c)Before trimming optimization

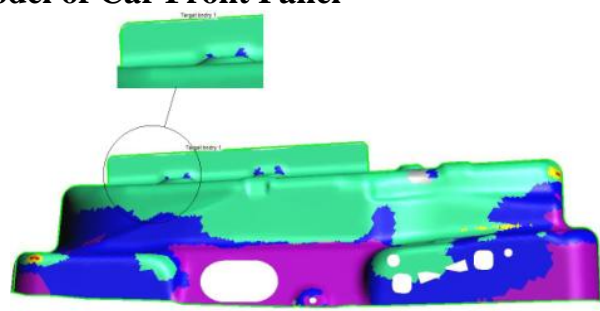

d)After trimming optimization

Fig.6 Before and After Optimization for Forming Plan of Car Front Panel

IV. WHOLE PROCESS SIMULATION PROCESS OPTIMIZATION

\section{A. Cracking Optimization}

Cracking is due to insufficient strength or plasticity of the material. It will occur when tensile stress exceeds the critical value. There are two forms of cracking, one is the cracking under biaxial tension stress, the other is the cracking under tension stress, which is easier to crack than the latter, but the maximum shear stress always plays an inductive role in the cracking process. Under the maximum shear stress, stress concentration is generated and eventually cracking.

There are five kinds of cracking optimization methods ${ }^{[9]}$ : shape optimization, drawing rib optimization, sheet contour optimization, process orifice and notch setting optimization and forming process optimization. Forming diagram and forming limit diagram FLD before cracking optimization are shown in Fig.6(a). Stress and strain point clouds crack through forming limit curve FLC and curved .The cracking position is shown by the forming drawing. In view of this cracking phenomenon, the specific method of enlarging the local roundness of the product is applied to 
shape optimization.. Add chamfering in MOD, select three chamfering corners in area A of parts, change radius $10 \mathrm{~mm}$, $10 \mathrm{~mm}, 10 \mathrm{~mm}$ to $15 \mathrm{~mm}, 25 \mathrm{~mm}, 20 \mathrm{~mm}$ respectively, and update all process settings. After checking and submitting the calculation, the optimized forming drawing and thickness analysis drawing are shown in Fig. 6 (b). The cracking phenomenon in area B of the part is obviously improved, and the thickness of the cracking area rises from $0.48 \mathrm{~mm}$ to $0.98 \mathrm{~mm}$.

\section{B. Edge cutting optimization}

Due to the plastic deformation of the part after drawing, Trim trimming optimization is usually used to simulate the multi-sampling process in the field. The product boundary line is defined as the target boundary, and the product boundary after drawing is approached continuously by iteration calculation through Trim trimming line optimization until the required product boundary tolerance is reached. Fig. 6 (c) shows that the actual boundary contour of the workpiece has a large error with the target boundary contour. A trim item is created on the settings page of Draw20 drawing process. The trim line is associated with the boundary contour of the right part of the target part. It is defined as 3D trimming type. The maximum number of iterations is set to 5 times and the maximum deviation is $0.5 \mathrm{~mm}$. The operation is resubmitted. The optimized trimming forming drawing is shown in Fig. 6 (d).

\section{CONCLUSION}

Through the analysis of the forming process of the right part of the car front panel, the whole process plan is planned as OP05 blanking, OP10 primary drawing, OP20 secondary drawing, OP30 trimming and punching, OP40 flanging. According to the whole process plan, the whole process multi-step stamping forming simulation is made. The simulation results show that cracking occurs at the corner of a product, and the error between the actual boundary profile and the target boundary profile of the product is large.

Aiming at the whole forming process of the parts, the corresponding optimization of forming process was formulated, and the cracking problem of the parts was eliminated by the optimization of product shape. By optimizing the trimming line, the error between the actual and objective boundary contour of the part is reduced. The optimized part meets the requirements of forming quality and precision, and seamless butt-joint between simulation and optimization of multi-step stamping process is realized.

\section{ACKNOWLEDGMENT}

This work was supported by the key projects of natural science research in Anhui province higher education institutions, (Project number:KJ2017A896) and Anhui Provincial Academic (Professional) top-notch talents academic funding project, The first author acts as responsible person (gxbjZD63)

\section{REFERENCES}

[1] H. Zhao-ming, WANG Li,LIU Xiao-fei,ZHANG Cheng. Numerical Simulation of Multi-position Progressive Stamping Based on Autoform Software[J].Journal of Hefei University of Technology (Natural Science),2015,38(2):157-160.
[2] Z. Min Xiong HegenLi Gui Wang Wensheng Chen Zhiping. Numerical Simulation of Whole process For multi-station Progressive Die of Complex Automotive Structure Parts[J].Forging \& Stamping Technology,2017,42(12):31-36.

[3] H. Zhao-ming, ZHAO Heng-wen, PAN Jin-yuan. Numerical Simulation of Multi Station Progressive Stamping Forming Linkage[J].Journal of Shandong University of Technology(Natural Science Edition), 2018,32(04):35-39.

[4] H. Zhao-ming, ZHAO Heng-wenLIU Xiao-fei, ZHANG Cheng. Simulation and Optimization for Multiple Drawing and Inside-out Redrawing of Cylindrical Parts[J].Journal of Nanjing Institute of Technology(Natural Science Edition),2018,16(01):44-48.

[5] C. Xue-jian, HUANG Zhao-ming, WANG Li, HU Li-yong. A CAE Driven of Drawing Process Surface Design for Car Beam[J]. Journal of Changchun Institute of Technology (Natural Science Edition),2018,19(01):44-48.

[6] M. Merkel, Schumacher A. An Automated Optimization Process for a CAE Driven Product Development[J]. Journal of Mechanical Design, 2003, 125(4):694-700.

[7] X. Zhou, Di-Sheng O U. Drawing Die Design of Automobile Floor Lateral Beam based on CAD/CAE $[J]$. Forging \& Stamping Technology, 2013.

[8] A N. Zongquan, Huang Z, Wang L. Simulation and Optimization of Drawing Process for Radiator Cross Beam[J]. Journal of Jiujiang University, 2017.

[9] D. Anindya , Ajit Naravane, Clifford C. Chou. A Practical CAE-Driven Approach for Weight Optimization of an Existing Vehicle Body[J]. 2006, submitted for publication.

[10] W Xiong, Y Yang, Liu H. Numerical simulation and optimization of stamping forming process for bracket based on orthogonal experiment method[J].China Metalforming Equipment \& Manufacturing Technology, 2016.

[11] Jae-Jun Lee,Gyung-Jin Park. Optimization of the structural and process parameters in the sheet metal forming process[J].Journal of Mechanical Science and Technology,2014(2):605-619.

[12] Hu Wang, Enying Li, Guang Yao Li. Parallel boundary and best neighbor searching sampling algorithm for drawbead design optimization in sheet metal forming $[\mathrm{J}]$.Structural and Multidisciplinary Optimization,2010,41(2),309-324.

[13] Hyuk Jong Bong,Frédéric Barlat,Myoung-Gyu Lee,Deok Chan Ahn. The forming limit diagram of ferritic stainless steel sheets: Experiments and modeling[J].International Journal of Mechanical Sciences, 2012,64(1),1-10.

[14] Hagenah H, Merklein M, Lechner M, et al. Determination of the Mechanical Properties of Hot Stamped Parts from Numerical Simulations [J]. Procedia Cirp,2015,33:167-172.

[15] Moussa C, Bartier O, Hernot X, et al. Mechanical characterization of carbonitrided steel with spherical indentation using the average representative strain[J].Materials \& Design,2016,89:1191-1198.

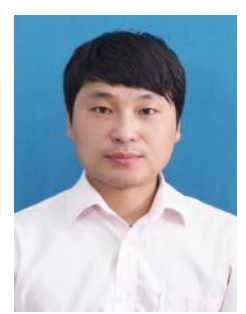

HUANG Zhao-ming: male, lecturer, master, graduated from HeFei University of Technology, vehicle engineering. His research direction is the theory and method of modern vehicle design. Now he is the vice president of Hohai University Wentian College, College of Mechanical Engineering. $\mathrm{He}$ is also deputy director of the Institute of mechanical engineering and director of the office of teaching and scientific research. Over the past five years, he has chaired or mainly participated in more than 10 key scientific research projects at the provincial level, published more than 30 papers of three types or above by the first author or communication author, and authorized more than 20 patents. Social positions related to scientific research: Director of Anhui Engineering Graphics Society, Member of Ma'anshan Science and Technology Association. 


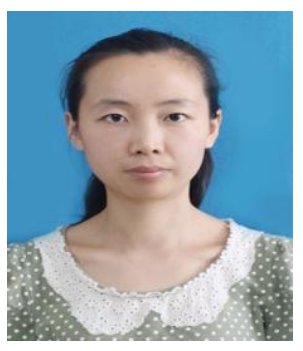

WANG Li: female, lecturer, master, graduated from Kunming University of Science and Technology, major in transportation engineering. She's research direction is the theory and method of modern vehicle design. Now she is in charge of automobile marketing and service in Xuancheng Vocational and Technical College. She guided students to participate in the provincial level two prize 1 , hosted 2 provincial-level topics, 2 edited textbooks. Scientific research: as the first author or correspondent author, 7 papers are published, and more than 10 patents are authorized. 